\title{
Effects of Unresolved High-Frequency Signals in Altimeter Records Inferred from Tide Gauge Data
}

\author{
Rui M. PONTE \\ Atmospheric and Environmental Research, Inc., Lexington, Massachusetts \\ FLORENT LyARD \\ LEGOS, Toulouse, France
}

12 April 2001 and 24 October 2001

\begin{abstract}
Rapid sea level signals are poorly resolved, or "aliased," by the TOPEX/Poseidon altimeter, due to its $\sim 10$ day repeat cycle (Nyquist period $T_{N} \sim 20$ days). To assess the potential for temporal aliasing in the altimeter data, the authors analyze hourly sea level records from a set of 110 island and coastal tide gauges. Time series are detided to focus on nontidal effects. Large variance is found at periods $<T_{N}$ (more than $50 \%$ of the total variance at high latitudes), with important contributions from the shortest periods $(<2$ days $)$. The inverted barometer correction reduces high-frequency variance at mid- and high latitudes but not at low latitudes. As inferred from the tide gauge analysis, in the worst-case scenario (one data point per altimeter repeat cycle), the ratio of aliased to measured variance can range from $\sim 0.1$ at the annual period to $\sim 0.8$ near $T_{N}$. Moderate data averaging (three points per altimeter repeat cycle, given a $\sim 3.3$-day near-repeat orbit) can substantially reduce the aliasing, however. The impact of aliasing is, in general, worse for coastal than for island stations.
\end{abstract}

\section{Introduction}

As is well known from sampling theory, records sampled at a given time interval $\Delta t$ cannot resolve signals with periods shorter than $2 \Delta t$-the so-called Nyquist period, $T_{N}$. The unresolved signals appear in the records as variability of longer periods and are said to be aliased. A main goal of any sampling strategy is, thus, to measure at sufficiently small $\Delta t$ to resolve all frequencies containing substantial power in order to minimize aliasing. If that is not possible, methods such as smoothing or modeling the unresolved signals are needed to mitigate the problem.

Since the inception of satellite altimetry, aliasing has been a prime concern in mission design (Parke et al. 1987) and data interpretation (Schlax and Chelton 1994; Chen and Ezraty 1997; Ray 1998), with primary focus on the large effects of the tides. As a result of the careful choice of orbit, data from the TOPEX/Poseidon (T/P) altimeter has led to vastly improved models of the tides, which are now routinely applied, with much success, to

Corresponding author address: Dr. Rui M. Ponte, Atmospheric and Environmental Research, Inc., 131 Hartwell Ave., Lexington, MA 02421-3126.

E-mail: ponte@aer.com removing tidal signals from the data. Besides the tides, there is a continuum of unresolved high-frequency (HF) sea level variability. Although initially downplayed (Parke et al. 1987), the impact of the HF continuum has slowly gained more attention (Chen and Ezraty 1997). Recent model results and data comparisons (Fukumori et al. 1998; Stammer et al. 2000; Tierney et al. 2000) have indicated that atmospherically forced HF barotropic signals can be a source of large aliasing in the T/P records at mid- and high latitudes. A more careful examination of the importance of the HF continuum thus seems in order.

One way of quantitatively assessing aliasing in altimeter records is to analyze in situ measurements with a higher sampling rate. In this note, we use an extensive set of tide gauges to examine the HF nontidal aliases. The in situ sea level records are directly relevant to the altimeter as demonstrated by numerous comparisons between the two types of data (e.g., Mitchum 1994). Our analysis of aliasing complements that of Ray (1998), who focused on tidal effects, and extends that of Gille and Hughes (2001), who analyze bottom pressure data in the same context. Although we focus on altimetry, results are also of interest to aliasing challenges facing gravity satellite missions, such as the Gravity Recovery 


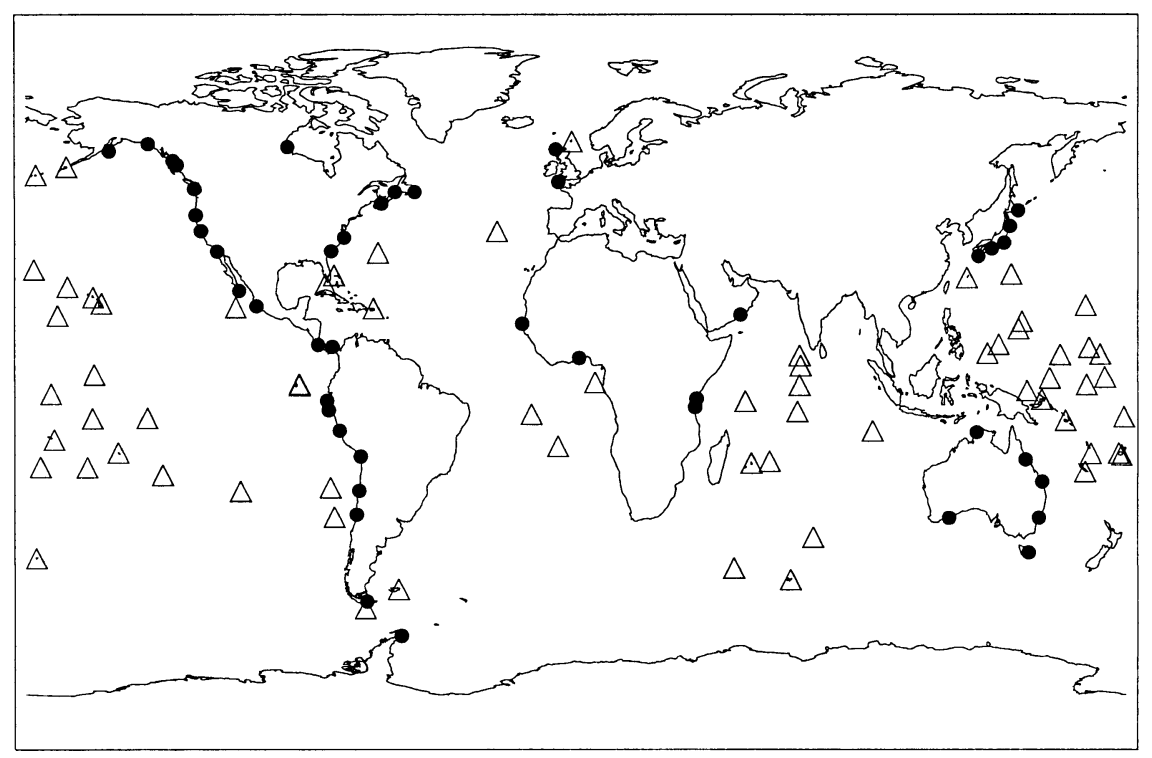

FIG. 1. Location of the 110 tide gauges used in the study. Coastal and island stations are denoted by solid circles and open triangles, respectively.

and Climate Experiment (GRACE) (Wahr et al. 1998). In the remaining sections, the data and methodology are described, followed by a presentation of our main analysis and results. Implications of our findings are discussed in the concluding section.

\section{Data and methodology}

The analysis is based on the set of 110 tide gauge records given in Fig. 1, which was assembled for other purposes (see Ponchaut et al. 2001) from the extensive archive maintained at the University of Hawaii's World Ocean Circulation Experiment (WOCE) Sea Level Center and the British Oceanographic Data Centre. Stations are spread over all oceans and latitudes, but with denser coverage in the tropical Pacific. Sixty-six island and 44 coastal stations are used. Each of these subsets is analyzed separately because island data can be more representative of the altimeter records (e.g., Mitchum 1994). Island and coastal results do not differ substantially; however, differences will be discussed where appropriate.

Given the focus on nontidal effects, we use detided series [see Ponchaut et al. (2001) for details on the detiding procedure]. The largest high-frequency tides, as well as the fortnightly and monthly tides, are removed from the original records. As with any detiding procedure, some residual tidal energy is inevitable. Although different methods may be more or less efficient at removing tidal signals (Ray 1998), the harmonic analysis method used by Ponchaut et al. (2001) to detide the hourly in situ data is likely better than the available detiding procedures applied to the altimeter data. Thus, any tidal residuals left in our analysis should conser- vatively mimic similar residuals in the altimeter records after tide corrections are implemented.

Besides detiding, common practice in altimeter data processing also involves the use of the inverted barometer (IB) correction (e.g., Ponte 1993). For the purpose of assessing the value of such correction in reducing aliasing, we use surface atmospheric pressure fields from the operational products of the European Centre for Medium-Range Weather Forecasts (ECMWF). The 6-hourly ECMWF analyses on a $1.125^{\circ} \times 1.125^{\circ}$ global grid are bilinearly interpolated in space and linearly interpolated in time to each tide gauge location and sampling time. The IB correction is calculated as proposed by Ponte (1993), with time variations in the averaged pressure over the ocean taken into account.

The detided sea level time series, either with or without the IB correction applied, consist of 1-hourly values over the period 1985-98, but not all records span the entire 14 years, as they have different start and end dates. In addition, most time series have missing values. Simple linear interpolation is used to fill the gaps, but no extrapolation is done to extend records at the end points. These interpolated series are used in all results presented here.

\section{Assessing aliasing effects}

The study has been done with T/P and other similar altimeters in mind. The T/P repeat cycle is 9.9156 days, which gives $T_{N} \sim 20$ days. Thus, for our purposes, HF variability will refer to signals at periods $<20$ days. For satellites with longer repeat cycles (e.g., $\sim 17$ days for a Geosat follow-on and $\sim 35$ days for the European Remote Sensing Satellites ERS-1 and ERS-2), aliasing 

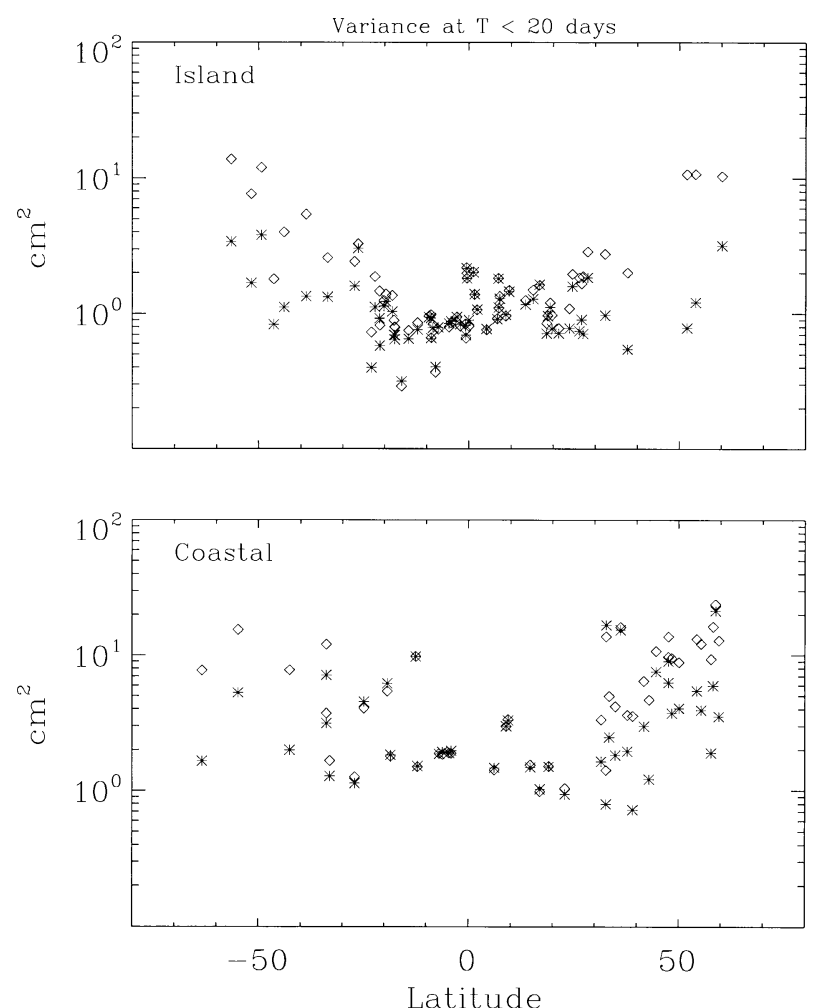

FIG. 2. Variance at periods $<20$ days. Each tide gauge is plotted at its respective latitude. Island and coastal stations are shown separately. Stars and diamonds represent results obtained with and without the IB correction applied, respectively.

problems are, in principle, expected to be more severe. However, longer repeat cycles usually mean denser spatial coverage. Thus, there is more data available for averaging, which may compensate somewhat for the coarser time sampling.

A straightforward measure of the HF variance is obtained by calculating the variance of the hourly records and subtracting from it the variance of 10-day averaged records, which approximately contain only variability with $T>20$ days. Boxcar averaging was used for simplicity. Some leakage of HF signals is inevitable in the averaged series, and the calculated HF variance values may be slightly underestimated. Similar effects may result from the linear interpolation used to fill the short gaps in the records. Thus, our estimates of HF variance can be taken as conservative. Values for island and coastal tide gauges, with and without the IB correction applied, are shown separately in Fig. 2 .

Results show a tendency for larger HF variability with latitude, particularly in the raw records, which is consistent with the increase in HF atmospheric forcing with latitude. The IB correction substantially reduces HF variances at mid- and high latitudes but not at low latitudes. In addition, the positive impact of the IB correction is somewhat larger at island stations. Results suggest a response to HF pressure forcing that deviates more from
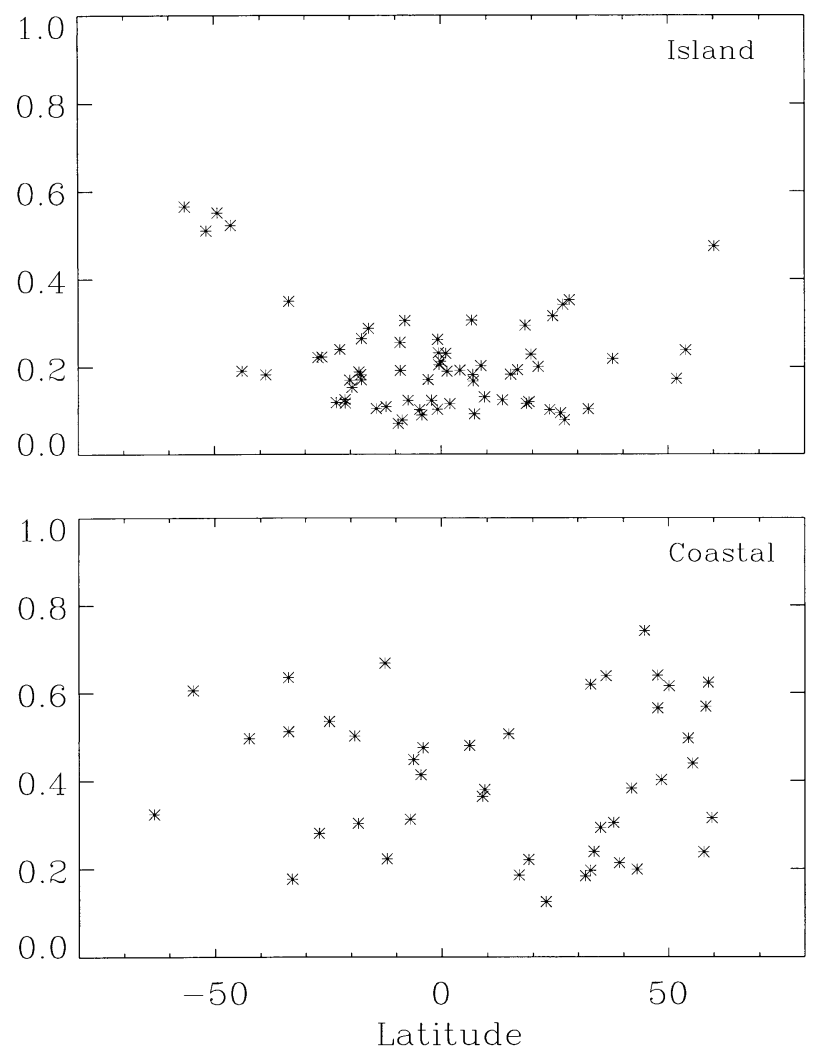

FIG. 3. Ratio of variance at $T<20$ days to total variance in the records, based on IB-corrected time series. Island and coastal stations are shown separately at each respective latitude.

IB behavior at low latitudes and coastal regions, consistent with previous findings (e.g., Ponte 1993; Ponte and Gaspar 1999). With IB effects removed, HF variances for island gauges range from values as low as 3 $\mathrm{cm}^{2}$ in the Tropics to more than $30 \mathrm{~cm}^{2}$ at high latitudes. Similar range is seen for coastal records, but with substantially higher values in general.

Aliasing effects will depend on how much of the total variance is related to HF signals. The ratio of the HF variance to the total variance is shown in Fig. 3. (Only results based on IB-corrected time series are shown in the rest of this paper, given the positive impact of the correction at mid- and high latitudes.) High-frequency variance can contribute more than $50 \%$ of the total variance at high-latitude island gauges, but more typical values range from $10 \%$ to $40 \%$. Values for coastal records are generally higher and show more spread with latitude. Contributions of the most rapid signals, including tidal residuals, to the HF variance are analyzed in Fig. 4, which shows the ratio of the variance at $T<$ 2 days, estimated using the same boxcar averaging method as before, to the variance at $T<20$ days. Signals with $T<2$ days indeed contribute substantially (more than $30 \%$ in most cases) to HF variance, especially at low latitudes where they can be dominant at places. 

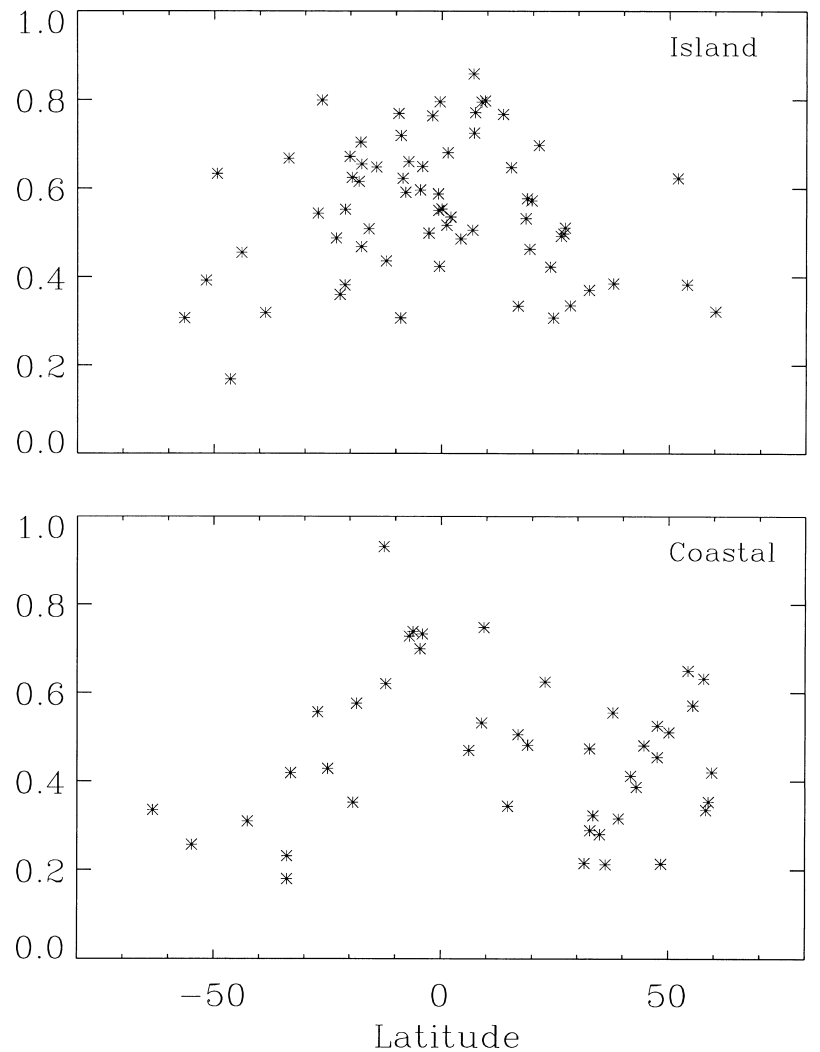

FIG. 4. As in Fig. 3, but for the ratio of variance at $T<2$ days to variance at $T<20$ days.

More generally, for most records more than $50 \%$ of the HF variance is contained at $T<6$ days (not shown).

Judging from the above results, the potential for aliasing in altimeter records is large at all latitudes. To help guide the analysis and interpretation of the altimeter records, it is useful to quantify, using simple spectral analysis, the amount of observed variance as a function of frequency that may be due to aliasing. To be specific, we assume that most altimeter data analyses seek to work with values representing the local averaged sea level for each $\sim 10$-day repeat cycle. Thus, we base our analysis on time series averaged over $238 \mathrm{~h}$ or 9.9167 days, which is closest to the T/P repeat cycle.

Three different averaged time series are used. The "true" time series, without aliased variability, is calculated by averaging all the 238 data points over each $\sim 10$-day sampling cycle. The other two "aliased" time series are meant to represent possible altimeter sampling scenarios. In a worst-case scenario, only one sample is present over each repeat cycle. To mimic this case, we use only one data point in each 238-h window to represent the time average in the second series. In most cases, however, altimeter data within the same repeat cycle can be averaged. In particular, given the $\sim 3.3-$ day near-repeat altimeter orbit, three data points may be available to construct the time average. Thus, a third time series is calculated by averaging three points at 2.3-day intervals within each window.

Each 10-day averaged series is divided into 1-yr segments, and the periodograms for each segment are calculated and averaged to estimate the variance at each frequency in the range 1-18.4 cycles per year (cpy). Given the arbitrary choice of data points in creating the averaged series in the aliased cases, series based on all possible choices are used, and respective periodograms are averaged. Subtracting the true periodogram from periodograms based on aliased series and dividing by the latter, one can calculate the percentage of "observed" variance due to aliasing. As each station has its own spectral characteristics, aliasing effects can vary from place to place. Rather than show a few examples, Fig. 5 plots mean results for island and coastal stations. Standard deviations are shown to give an idea of the range of behaviors from station to station.

As expected from purely theoretical arguments (e.g., Chen and Lui 2000), in all cases the effects of aliased energy are strongest at periods near $T_{N}$, for which half or more of the observed variance is due to aliasing and decay toward longer periods. Frequency dependence is rather smooth, but there is some evidence for enhanced aliasing near $6 \mathrm{cpy}$, close to the aliased frequencies for $\mathrm{M}_{2}$ and $\mathrm{S}_{2}$ tides (e.g., Ray 1998). The impact of aliasing is larger for coastal records in general. For the worst aliasing case of a single data point per repeat cycle, mean aliased variance is $>20 \%(40 \%)$ of the measured variance in island (coastal) stations for frequencies $>3$ cpy, and noticeable contamination can extend even to the annual cycle. Comparisons between the two aliased cases show that small amounts of data averaging can reduce the effects of aliasing substantially. For the threepoint averages used, the percentage of observed variance due to aliasing drops below $20 \%$ for frequencies $<6$ cpy. Submonthly timescales are, however, still very much corrupted.

\section{Concluding remarks}

Based on our tide gauge analysis, for altimeter applications that require along-track pointwise measurements with no data averaging involved, aliasing problems can significantly compromise results, even at the lowest frequencies. In general, at $T<4$ months, more than $20 \%$ of the observed variance can be due to aliasing. In altimeter studies where multiple-track data averaging is used, impact of aliasing should be much reduced, with contributions from aliased variance at $T$ $>2$ months likely to be below $20 \%$. The contamination at shorter periods is, however, likely to remain substantial even when data averaging is applied, making the study of such periods difficult.

Our results are generally in good agreement with those of Gille and Hughes (2001), inferred from analysis of bottom pressure data, and those of Ray (1998), based on three tide gauges. Ray (1998) apparently found al- 

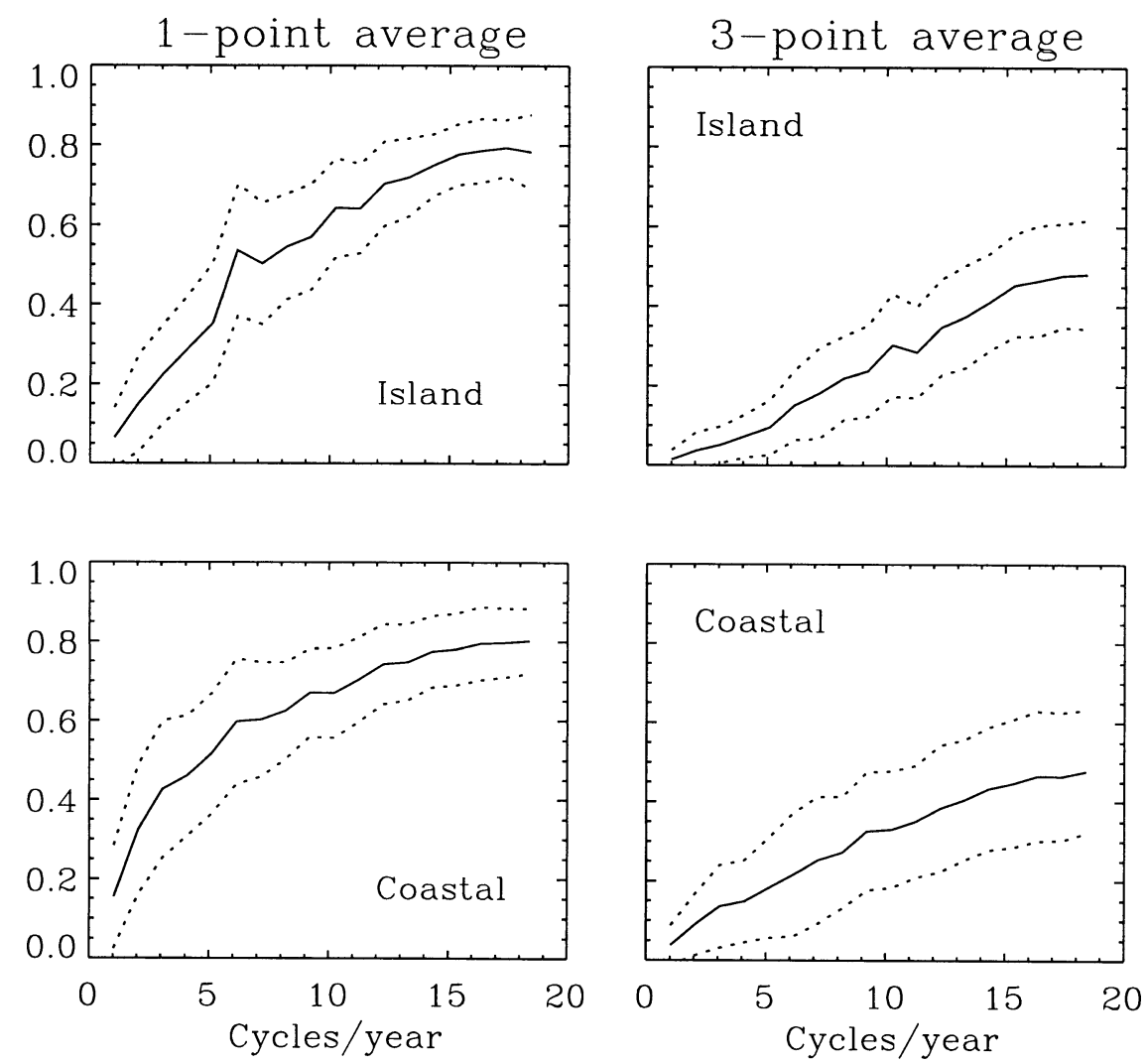

FIG. 5. Ratio of the aliased variance to the observed variance as a function of frequency, based on $\sim 10$-day averaged time series as described in the text. Results in left (right) panels assume that only one (three) data points are available in each $\sim 10$-day window to evaluate the aliased series. Mean results for island and coastal records are shown separately. The envelope curves (dotted) represent mean values \pm one std dev.

iasing effects on the annual cycle to be negligible. His results may have been influenced, however, by the strong amplitude of the annual cycle for the stations examined. Thus, it is not clear from his Figs. 7-9 what the effects of aliasing really are in percentage terms. Results for the worst aliasing case in our Fig. 5 indicate that, on the mean, $5 \%-15 \%$ of the observed variance at the annual period can be due to aliasing. The effect of aliasing at interannual periods is not addressed in Fig. 5 , but results at 1 cpy suggest that it could be important, as found by Ray (1998) for one of his stations.

Although the impact of aliasing can vary from place to place, one general trend can be discerned in our results. Coastal regions are likely to be more affected by aliasing. A variety of factors can contribute to this trend, and some are suggested by our analysis. Besides having generally enhanced HF variability, coastal regions may have more complicated tidal dynamics (e.g., Ray 1998) as well as a more dynamic response to pressure forcing (e.g., Ponte 1993), leading to a more difficult removal of these signals from the data. Dealing with these issues will be a challenge in the years ahead, particularly as coastal applications for altimeter data become more common.
In the interest of providing a wider range of applications to altimeter users, modeling or filtering methods should be used whenever possible to mitigate the aliasing effects in the data. Modeling of HF signals that are directly driven by surface atmospheric winds and pressure seems particularly promising (Stammer et al. 2000; Tierney et al. 2000; Hirose et al. 2001). Given the importance of aliasing contributions from variability at $T<2$ days, the need to deal with the most rapid signals cannot be dismissed. In this regard, improvements in tide models will continue to be crucial, especially in coastal regions, if one is to reduce the impact of tidal residuals in the data. Besides pure modeling efforts, data-based methods such as objective analysis can also be specifically designed to minimize aliasing effects (P.-Y. Le Traon 2000, personal communication). Ultimately, optimal estimation techniques that combine dynamic models and observations should prove most fruitful in improving our ability to estimate and understand both high- and low-frequency oceanic signals in the altimeter data.

Finally, we note that rapid sea level signals can have a strong barotropic component (e.g., Stammer et al. 2000), especially away from low-latitude regions, and 
thus be directly related to changes in ocean bottom pressure or mass. As such, the aliasing effects highlighted here will also impact gravity measurements from GRACE. The sampling characteristics and nature of the GRACE measurements are, however, very different from those typical of altimetry (Wahr et al. 1998). An assessment of aliasing impacts on GRACE might be more easily obtained from synthetic global ocean model data rather than a few point measurements, as used here.

Acknowledgments. Some of the references listed were brought to our attention by S. Jayne and R. Ray. This work was initiated during an extended visit of R. P. to LEGOS, made possible with support from the Observatoire Midi-Pyrénnées. Support for work at AER is provided by Contract 1206432 with the Jet Propulsion Laboratory under the Jason-1 project of NASA.

\section{REFERENCES}

Chen, G., and R. Ezraty, 1997: Non-tidal aliasing in seasonal sealevel variability and annual Rossby waves as observed by satellite altimetry. Ann. Geophys., 15, 1478-1488.

, and H. Lui, 2000: The effect of temporal aliasing in satellite altimetry. Photogramm. Eng. Remote Sens., 66, 639-644.

Fukumori, I., R. Raghunath, and L.-L. Fu, 1998: Nature of global large-scale sea level variability in relation to atmospheric forcing: A modeling study. J. Geophys. Res., 103, 5493-5512.

Gille, S. T., and C. W. Hughes, 2001: Aliasing of high-frequency variability by altimetry: Evaluation from bottom pressure recorders. Geophys. Res. Lett., 28, 1755-1758.

Hirose, N., I. Fukumori, V. Zlotnicki, and R. M. Ponte, 2001: Highfrequency barotropic response to atmospheric disturbances: Sensitivity to forcing, topography, and friction. J. Geophys. Res. 106, 30 987-30 995

Mitchum, G., 1994: Comparison of TOPEX sea surface heights and tide gauge sea levels. J. Geophys. Res., 99, 24 541-24 553.

Parke, M. E., R. H. Stewart, D. L. Farless, and D. E. Cartwright, 1987: On the choice of orbits for an altimetric satellite to study ocean circulation and tides. J. Geophys. Res., 92, 11 693-11 707.

Ponchaut, F., F. Lyard, and C. Le Provost, 2001: An analysis of the tidal signal in the WOCE sea level dataset. J. Atmos. Oceanic Technol., 18, 77-91.

Ponte, R. M., 1993: Variability in a homogeneous global ocean forced by barometric pressure. Dyn. Atmos. Oceans, 18, 209-234

__ , and P. Gaspar, 1999: Regional analysis of the inverted barometer effect over the global ocean using TOPEX/POSEIDON data and model results. J. Geophys. Res., 104, 15 587-15 601.

Ray, R., 1998: Spectral analysis of highly aliased sea-level signals. J. Geophys. Res., 103, 24 991-25 003.

Schlax, M. G., and D. B. Chelton, 1994: Detecting aliased tidal errors in altimeter height measurements. J. Geophys. Res., 99, 12 60312612.

Stammer, D., C. Wunsch, and R. M. Ponte, 2000: De-aliasing of global high frequency barotropic motions in altimeter observations. Geophys. Res. Lett., 27, 1175-1178.

Tierney, C., J. Wahr, F. Bryan, and V. Zlotnicki, 2000: Short-period oceanic circulation: Implications for satellite altimetry. Geophys. Res. Lett., 27, 1255-1258.

Wahr, J., M. Molenaar, and F. Bryan, 1998: Time variability of the earth's gravity field: Hydrological and oceanic effects and their possible detection using GRACE. J. Geophys. Res., 103, 30205 30229 . 